\title{
Technical refinements of bile duct division in living donor liver surgery
}

Mitsuhisa Takatsuki, Susumu Eguchi, Kosho Yamanouchi, Masaaki Hidaka, Akihiko Soyama,

Takashi Kanematsu

Department of Surgery, Nagasaki University Graduate School of Biomedical Sciences, Nagasaki, Japan

Short running title: Bile duct division in living donor hepatectomy

Key words: liver transplantation, living donor, bile duct

Address correspondence to:

Mitsuhisa Takatsuki, M.D.

Department of Surgery, Nagasaki University Graduate School of Biomedical Sciences, 1-7-1 Sakamoto,

Nagasaki 852-8501, Japan

TEL: 81-95-819-7316

FAX: 81-95-819-7319

E-mail: takapon@net.nagasaki-u.ac.jp 


\begin{abstract}
Background/purpose. In spite of the great risk involved, the donor bile duct division procedure has not been thoroughly addressed in the literature. The purpose of this study is to show the appropriate approach to bile duct division in living donor hepatectomy.

Methods. Of 87 living donor liver surgeries, we performed bile duct division by marking the cutting point using a small vascular clip under ordinary cholangiography in the first 37 cases, while the current procedure was used in 50 cases by encircling the cutting point using a radiopaque marker filament under real-time C-arm cholangiography.
\end{abstract}

Results. As regards the procurement of the 51 right lobe grafts, the incidence of multiple bile ducts in the graft was significantly reduced by our novel procedure (20/28 (71\%) vs. $7 / 23$ (30\%), $\mathrm{P}<0.01$, Fisher's test). Overall, there were no biliary strictures after surgery in any of the donors, with a median follow-up period of 43 months (range: 8-136).

Conclusions. Our procedure of bile duct division in living liver donor surgery enabled us to avoid the biliary stricture while cutting the bile duct of the donor with great accuracy. 
Living donor liver transplantation (LDLT) has been established as an effective modality for the treatment of various end-stage liver diseases. However, compared to deceased donor liver transplantation, more technical and ethical dilemmas exist, primarily because it is difficult to strike a balance between donor safety and recipient benefit. As regards biliary reconstruction in this context, the recipient requires a large, single bile duct orifice in order to reduce the risk of post-surgical biliary complications $(1,2)$. Thus, while it is desirable to cut the bile duct as close as possible to the hepatic hilum during donor surgery, this leads to significant concerns about biliary stricture in the donor. Donor safety should be the top priority in LDLT, and therefore the bile duct must be cut with great caution, and according to the most appropriate procedure. In spite of the great risk involved, the donor bile duct division procedure has not been thoroughly addressed in the literature. The aim of this study was therefore to describe our technical refinements to the procedure of bile duct division in living donor liver surgery.

\section{Patients and Methods}

\section{Patients}

Eighty-seven living donor hepatectomies for primary liver transplantation were performed at our institution from August 1997 to April 2008. The living donors consisted of 44 males and 43 females with a median age of 39 (range: 19-67). The following types of grafts were procured: 9 left lateral 
segments, 2 left lobes without the middle hepatic vein (MHV), 8 extended left lobes with the MHV, 12 extended left lobes with the caudate lobe, 51 right lobes without the MHV, and 5 right posterior segments. These cases were divided as follows into two groups according to the treatment period: group A (August 1997 to December 2004, n=37) and group B (January 2005 to April 2008, n=50) (Table 1). Preoperative evaluation of the biliary anatomy was performed by magnetic resonance cholangiopancreatography in both groups. In group A, we adapted a right lobe graft only for adult-to-adult LDLT, but thereafter we used various types of grafts (e.g., left liver grafts and right posterior segment grafts), primarily to enhance donor safety. Moreover, we altered the bile duct division procedure. Initially, we carried out ordinary cholangiography after the cholecystectomy, via a catheter which had been placed at the cystic duct, and the bile duct was cut prior to parenchymal transection by marking the cutting point using a small vascular clip (Fig 1). However, we frequently encountered cases with multiple bile ducts during right lobe graft procurement, such that we adapted the previous procedure to arrive at the current approach to bile duct division, as described below. This procedure is adapted in group B.

Surgical procedure of bile duct division

During hilar dissection, the gall bladder was dissected away from the liver, and the hepatic artery and portal branch were fully exposed and isolated from the hilar plate. Particular attention was paid 
to retaining the surrounding tissue of the hilar plate without exposing the bile duct; in order to avoid heat injury, electric cautery should not be used at this step. At the final step of the subsequent parenchymal transection, the hilar plate was fully exposed and encircled with a radiopaque marker filament obtained from surgical gauze (Fig. 2A). Real-time cholangiography using C-arm fluoroscopy was then performed via the catheter, which was placed in the cystic duct (Fig. 2B). To verify the optimal point for cutting the bile duct, the radiopaque filament was retracted (Fig. 2C), and the $\mathrm{C}$-arm was rounded to adjust the apparatus to the accurate angle. After confirmation of the accurate cutting point, parenchymal transection was further advanced using a liver hanging-maneuver technique (3) with preservation of the hilar plate. We then interposed the surgery after completion of the liver parenchymal transection. When the surgeon for the recipient required the graft, the hilar plate, including the hepatic duct, was precisely divided with scissors, and the stump of the remnant bile duct was closed with continuous, 6-0 absorbable, monofilament sutures (PDS II, Ethicon, Somerville, New Jersey). Cholangiography with C-arm fluoroscopy was then performed once again in order to check for biliary leakage and strictures in the remnant bile duct (Fig. 2D). The liver graft was then removed after the hepatic artery, portal vein, and hepatic vein were divided. 


\section{Biliary reconstruction in recipients}

Biliary reconstruction in the recipients was performed by Roux-en-Y hepaticojejunostomy or duct-to-duct anastomosis, with or without biliary stenting, and with interrupted, 6-0 absorbable, monofilament sutures (PDS II, Ethicon, Somerville, New Jersey) (Table 1). Duct-to-duct anastomosis was the first-line, and hepaticojejunostomy was performed only in the cases of biliary atresia, primary sclerosing cholangitis, and in the cases that the quality of recipient bile duct was not good with various reasons, such as biliary ischemia. In case with multiple bile ducts in the graft, duct plasty was performed whenever possible (4), and duct-to-duct anastomosis was still the first-line of biliary reconstruction. As decreasing the incidence of biliary atresia, the incidence of hepaticojejunostomy was significantly lower in group B (Table 1).

\section{Results}

Incidence of multiple bile ducts in grafts

The characteristics of the biliary anatomy increase the probability of multiple bile ducts in right lobe grafts over that in left lobe grafts ${ }^{4}$. The incidence of multiple ducts in right lobe grafts was compared between groups. In the procurement of 51 right lobe grafts, the incidence of multiple bile ducts was significantly reduced by the current procedure (20/28 (71\%) in group A vs. 7/23 (30\%) in group $\mathrm{B}, \mathrm{P}<0.01$, Fisher's test). In these cases, anatomic variation was similar in both groups, i.e., 
according to the classification system of Varotti et al. (5) (Fig. 2) (type 1 (right anterior and right posterior hepatic ducts (HD) join together to form the right HD: 19/28 (67.9\%) in group A vs. 17/23 (73.9\%) in group B), type 2 (the right $\mathrm{HD}$ is absent and the right anterior $\mathrm{HD}$ and right posterior HD join directly to the confluence with the left HD to form the common HD: 2/28 (7.1\%) in group A vs 3/23 (13.0\%) in group B), type 3 (the right anterior HD or the right posterior HD open directly into the left HD: 6/28 (21.4\%) in group A vs 3/23 (13.0\%) in group B), type 4 (the right anterior HD or the right posterior HD open directly into the common HD: 1/28 (3.6\%) in group A vs 0/23 (0\%) in group B)).

Bile duct division in complex cases

In cases involving multiple bile ducts in the graft, one hilar plate was encircled, including all of the hepatic ducts (Fig. 3). In these cases as well, the use of a radiopaque marker filament as the reference for the optimal cutting point was feasible, and allowed preservation of the surrounding tissue. In cases involving a right posterior segment graft, it can generally be relatively difficult to determine the optimal cutting point, because the targeted point tends to be more distal and supports smaller bile duct(s); however, in all of our 5 donor cases involving this type of graft, we easily identified the optimal cutting point without any difficulty (Fig. 4). In some cases involving a complex variation in the bile duct branching pattern (i.e., right posterior hepatic duct independently 
branching from the left hepatic duct), we were able to avoid bile duct injury in the donor by clearly

making the cutting point with pulling the radiopaque marker filament (Fig. 5).

Biliary complications in donors

A total of 16 complications (18.4\%) were seen in the donors (6 bile leakages, 3 wound infections,

2 pleural effusions, 2 cases of gastric stasis, 1 portal vein thrombosis, 1 case of postoperative bleeding, and 1 paralytic ileus). All bile leakage cases were treated by percutaneous drainage, and all spontaneously resolved without requiring surgical intervention. There was no significant difference in the incidence of bile leakage between the groups (4/37 (10.8\%) in group A vs 2/50 (4.0\%) in group B). No biliary strictures were observed in any of the donors in either of the groups. All donors are alive and currently doing well, carrying out normal daily activities after a median follow-up period of 43 months (range, 8-136).

\section{Biliary complications in recipients}

The incidence of biliary stricture requiring endoscopic or surgical treatment of recipients was compared between adult cases in both groups ( $>18$ years). It would not be appropriate to formally compare the incidence of biliary complications in recipients according to the groups as defined here, because the follow-up period for group B was significantly shorter than that for group A. However, it 
should be noted that the incidence of biliary stricture was non-significantly lower in group B than group A (i.e., 9/37 (24.3\%) in group A vs. 8/50 (16.0\%) in group B).

\section{Discussion}

Biliary stricture is one of the most significant complications in liver transplant recipients. The etiology of this complication is multifactorial, and especially in LDLT, the presence of tiny, multiple ducts can contribute to a higher incidence of biliary stricture than that encountered in deceased donor liver transplantation. Although the relationship between the presence of multiple ducts and the incidence/severity of biliary complication remains controversial $(6,7)$, several studies have indicated that the presence of multiple bile ducts in a graft is a risk factor for biliary complication $(1,2)$. Additionally, biliary ischemia is an important, well-documented factor that affects biliary stricture $(8,9)$. Considering these factors, surgical innovations are required not only in recipient surgeries, but also in donor surgeries. In order to maintain the blood supply, it is desirable to harvest a large bile duct orifice in the graft, together with a sufficient amount of surrounding tissue. However, there remain several technical and ethical dilemmas associated with this procedure. In particular, in order to obtain a graft containing a single large orifice, it is necessary to cut the bile duct as close as possible to the hepatic hilum, which can lead to biliary stricture in the residual bile duct of the donor. Additionally, in the attempt to cut the bile duct at a precise point, it becomes necessary to expose the 
bile duct by also dissecting back the surrounding tissue, which can lead to biliary ischemia. The technical innovations described in this study yielded a resolution to these problems. By encircling the hilar plate using a radiopaque marker filament, the cutting point was easily identified, and the surrounding tissue of the hilar plate could be maintained. Under real-time C-arm cholangiography while pulling the filament, the cutting point was clearly visualized in a 3-dimensional image. This procedure using a radiopaque marker filament and C-arm cholangiography was originally introduced by Chen in Taiwan (10). We then modified the procedure by pulling the filament in order to render the cutting point more clear, as described above. Initially, we adopted this approach for the procurement of right lobe grafts, due to the high incidence of multiple bile ducts encountered in these grafts (11). After the adoption of this novel technique, the incidence of encountering multiple ducts in grafts was significantly reduced without increasing the rate of biliary stricture in donors. Biliary stricture is one of the most significant complications in living donors. According to a survey conducted by the Japanese Liver Transplantation Society, 11\% of 1852 donors had biliary leaks and strictures, the majority of which occurred after right-lobe hepatectomy; a total of 10 donors underwent surgical revision for biliary complications (12). To date, we have not observed any biliary complications requiring surgical treatment among our donors. Six cases (7.7\%) exhibited minor bile leakage, which in each case was successfully treated by percutaneous drainage alone, i.e., no subsequent biliary strictures developed in any of these donors. With the increasing rate of adult 
LDLT, several types of graft have been introduced (e.g., left lobe with or without the caudate lobe, right posterior segment graft). In all cases presented here, our novel approach was found to be very effective at enhancing the accuracy of setting the cut point for resection of the bile duct.

One disadvantage of this technique could be possible bile duct injury during encirclement of the hilar plate. Thus, very careful attention should be taken at this step to notice any resistance and thus to avoid forced penetration of the Kelly clamp into the fibrous tissue. Furthermore, it remains important to obtain clear preoperative visualization of the biliary anatomy, thereby to avoid missing any significantly aberrant branching pattern around the hilum. Here, we used magnetic resonance cholangiopancreatography for the preoperative evaluation of the biliary anatomy, and no significant bile duct injuries occurred. In general, the key point of the present procedure is that the hilar plate (i.e., not the bile duct) should be fully exposed before it is marked with the radiopaque marker filament. Thus, the hilar anatomy should be clearly visualized, including assessment of the relationship between the hepatic artery, the portal vein, and the hilar plate. As long as the tissue of hilar plate is preserved, no significant biliary ischemia is likely to occur. Once the encirclement of the hilar plate was completed, this procedure was found to be useful even in cases involving complex bile duct branching patterns. Intraoperative cholangiography just prior to the encirclement of the hilar plate may be helpful in yielding the most secure bile duct division possible.

Whereas donor safety should be the top priority in LDLT, the recipient's outcome is almost 
equally important in terms of rewarding the donor for his or her devotion to the patient. Our current procedure contributed to a reduction in the incidence of biliary stricture in recipients as well. Randomized and controlled studies would be ideal, but until we have access to such studies, we believe that harvesting a single orifice with sufficient surrounding tissue of bile ducts is a feasible means of performing the most straightforward surgery possible, and this novel approach is expected to contribute to successful outcomes. Moreover, simple anastomosis using a single orifice might facilitate the treatment of any remaining cases involving biliary stricture.

In conclusion, the present procedure of dividing the bile duct during living-donor liver surgery using a radiopaque marker filament and C-arm cholangiography is feasible for avoiding biliary stricture in the donor, while maintaining graft quality with sufficient surrounding tissue and reducing the chance of encountering multiple bile duct orifices. 


\section{References}

1. Testa G, Malagó M, Valentín-Gamazo C, et al.

Biliary anastomosis in living related liver transplantation using the right liver lobe: techniques and complications. Liver Transpl 2000 ;6: 710-4.

2. Gondolesi GE, Varotti G, Florman SS, et al. Biliary complications in 96 consecutive right lobe living donor transplant recipients. Transplantation 2004; 77: 1842-8.

3. Belghiti J, Guevara OA, Noun R, et al.

Liver hanging maneuver: a safe approach to right hepatectomy without liver mobilization.

J Am Coll Surg 2001; 193: 109-11.

4. Fan ST, Lo CM, Liu CL, et al. Biliary reconstruction and complications of right lobe live donor liver transplantation. Ann Surg. 2002; 236: 676-83.

5. Varotti G, Gondolesi GE, Goldman J, et al.

Anatomic variations in right liver living donors. J Am Coll Surg 2004; 198: 577-82.

6. Ishiko T, Egawa H, Kasahara M, et al. Duct-to-duct biliary reconstruction in living donor liver transplantation utilizing right lobe graft. Ann Surg 2002; 236: 235-40.

7. Liu CL, Lo CM, Chan SC, Fan ST. Safety of duct-to-duct biliary reconstruction in right-lobe live-donor liver transplantation without biliary drainage. Transplantation 2004; 77: 726-32. 
8. Northover JM, Terblanche J. A new look at the arterial supply of the bile duct in man and its surgical implications. Br J Surg 1979; 66: 379-84.

9. Yanaga K, Sugimachi K. Biliary tract reconstruction in liver transplantation.

Surg Today. 1992;22:493-500.

10. Chen CL. Bile Duct Reconstruction: Proposal from Asian Experience. $10^{\text {th }}$ Annual Congress of the International Liver Transplantation Society, June 9-12, 2004, Kyoto, Japan.

11. Takatsuki M, Eguchi S, Tokai H, et al. A secured technique for bile duct division during living donor right hepatectomy. Liver Transpl 2006; 12: 1435-6.

12. Umeshita K, Fujiwara K, Kiyosawa K, et al; Japanese Liver Transplantation Society. Operative morbidity of living liver donors in Japan. Lancet 2003; 362: 687-90. 


\section{Figure legend}

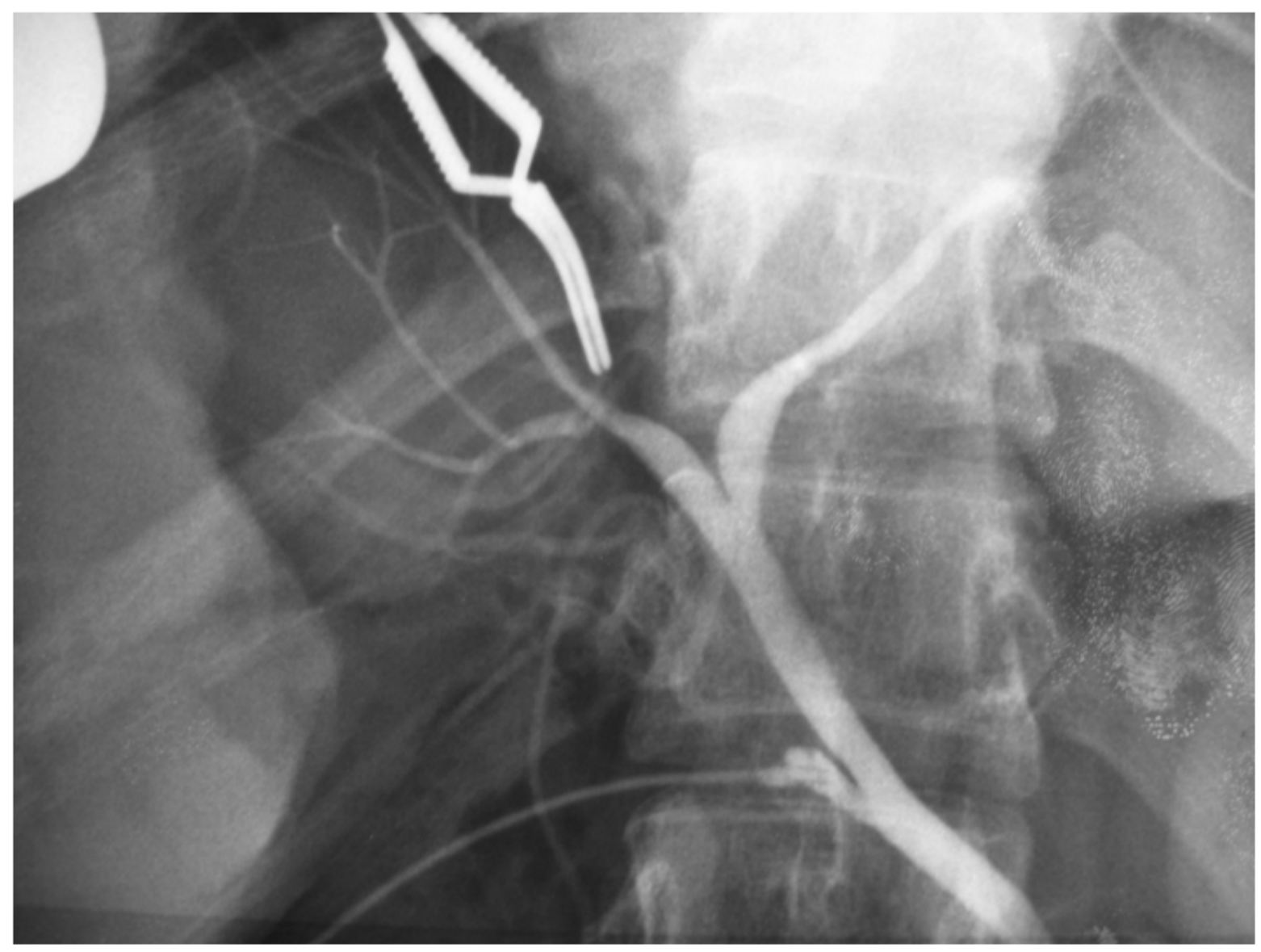

Figure 1

Our original procedure for bile duct division. The bile duct is divided under ordinary

cholangiography by placing a small vascular clip around the hepatic duct. 


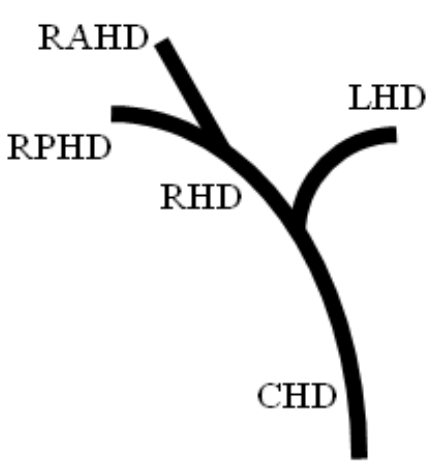

Type 1

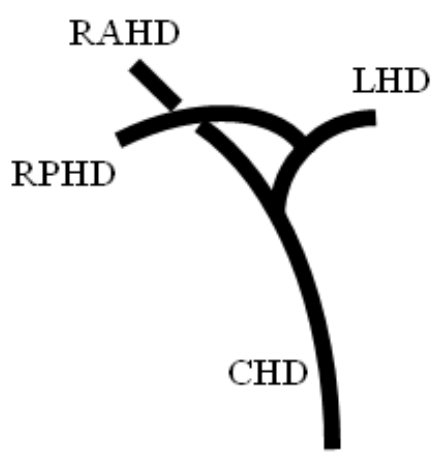

Type 3b

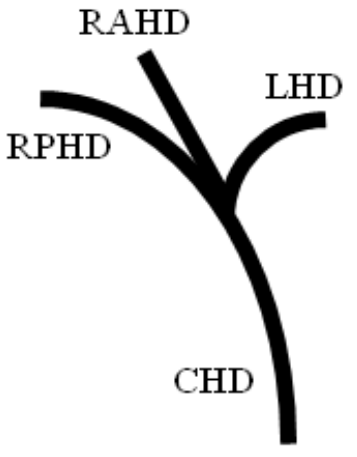

Type 2

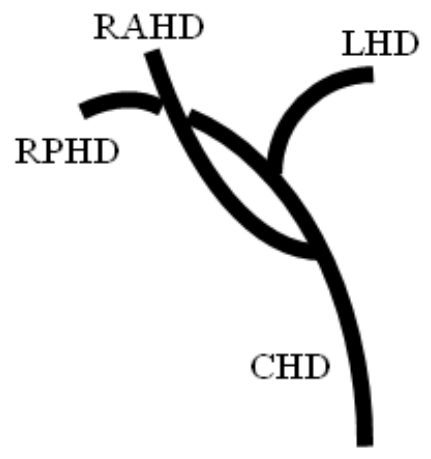

Type 4a

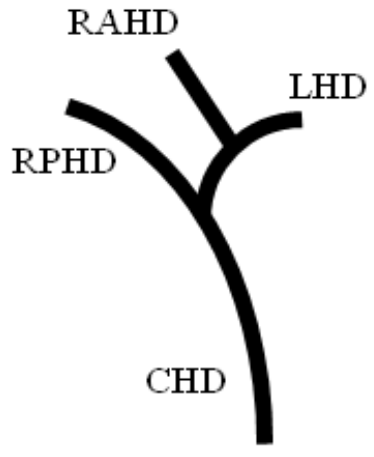

Type 3a

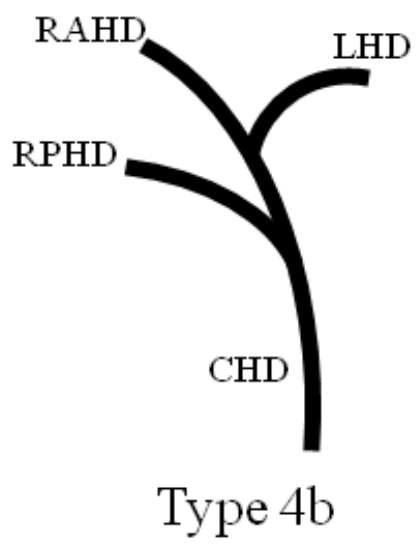

Figure 2

Classification of the biliary tree anatomy by Varotti et al.(5). CHD, common hepatic duct; LHD,

left hepatic duct; RAHD, right anterior hepatic duct; RPHD, right posterior hepatic duct. 


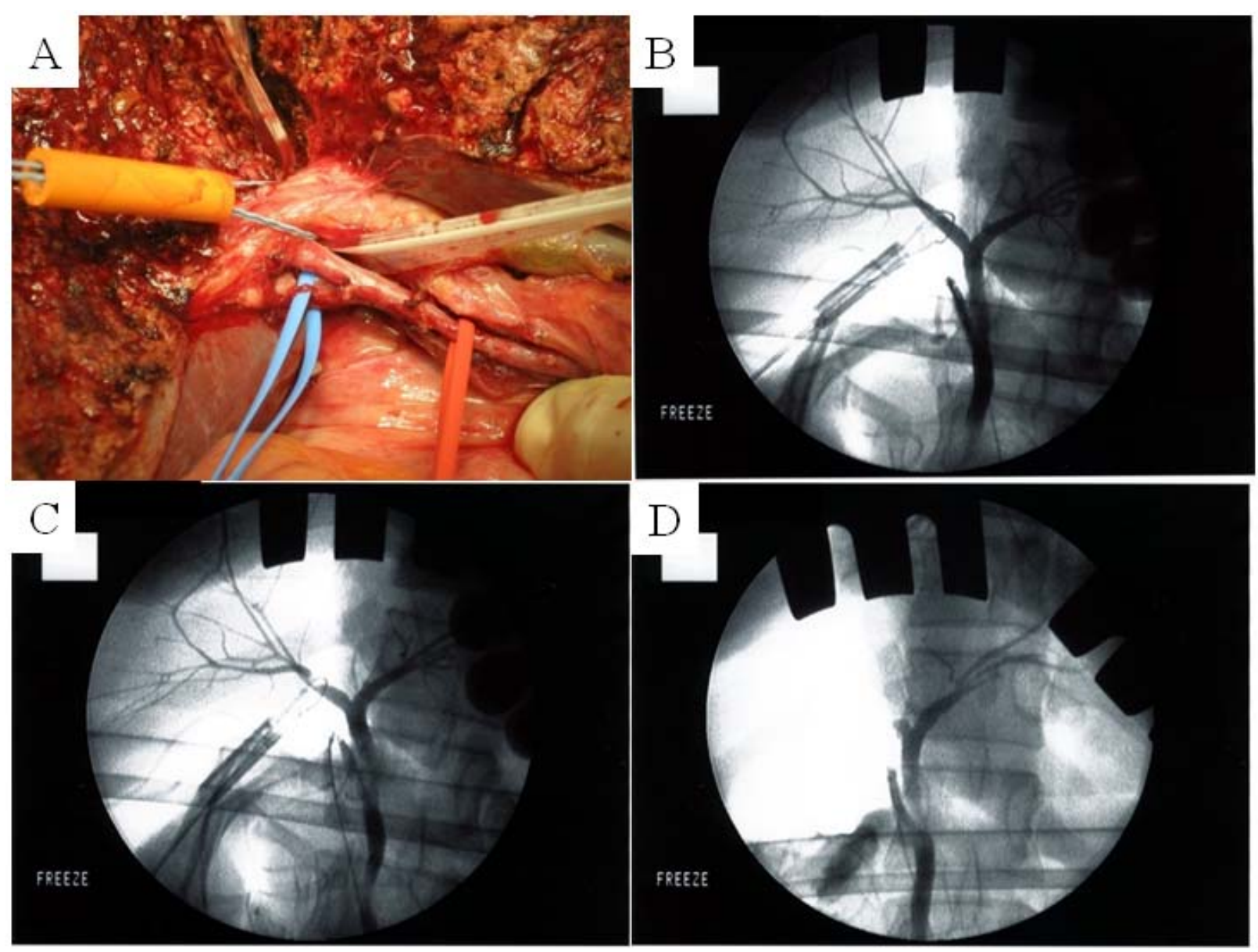

Figure 3

Bile duct division in the procurement of a right lobe graft. The right hilar plate is encircled with a

radiopaque marker filament, and sufficient surrounding tissue is preserved, without exposure of

the bile duct (A). C-arm cholangiography revealed that the radiopaque marker filament was placed

at an adequate point at the right hepatic duct (B). The radiopaque marker filament was pulled in

order to verify the cutting point (C). C-arm cholangiography after bile duct division revealed that

the bile duct was cut at the optimal point, without inducing a stricture in the remnant left hepatic

duct (D). 


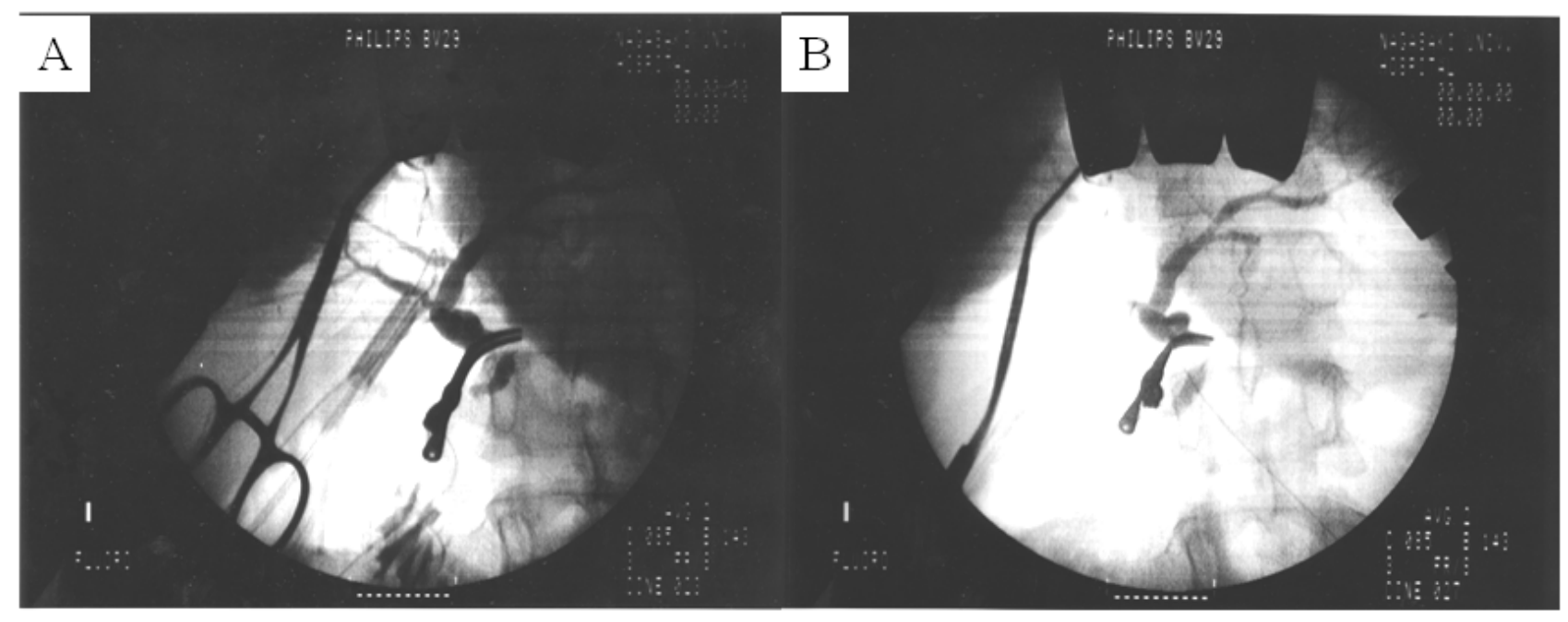

Figure 4

Bile duct division in the procurement of a right-lobe graft with multiple ducts. The right hilar plate,

including both the right anterior branch and the right posterior branch, is encircled by a

radiopaque marker filament (A). There was no stricture in the remnant left hepatic duct after

division (B). 


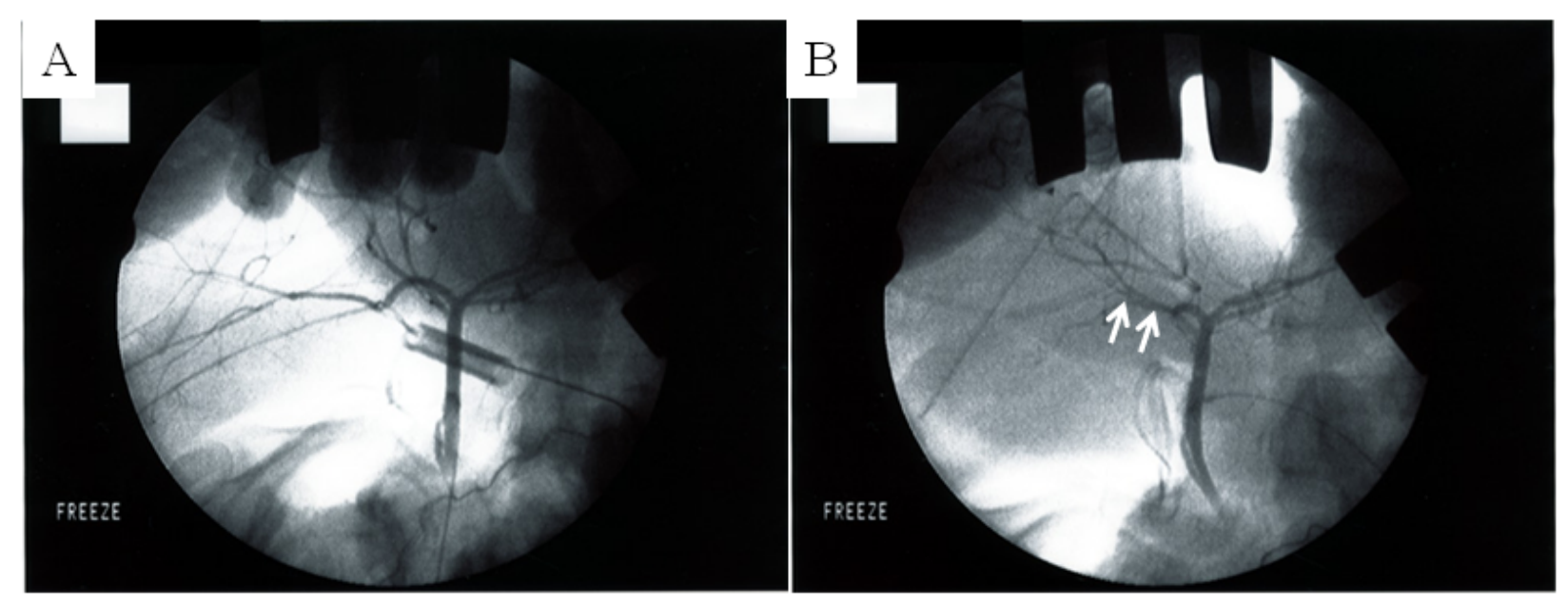

Figure 5

Bile duct division in the procurement of a right posterior segment graft. A radiopaque marker

filament was placed at an adequate point in the right posterior hepatic duct (A). After division of

the right posterior hepatic duct, there was no stricture in the remnant right anterior hepatic duct

(arrow, B). 


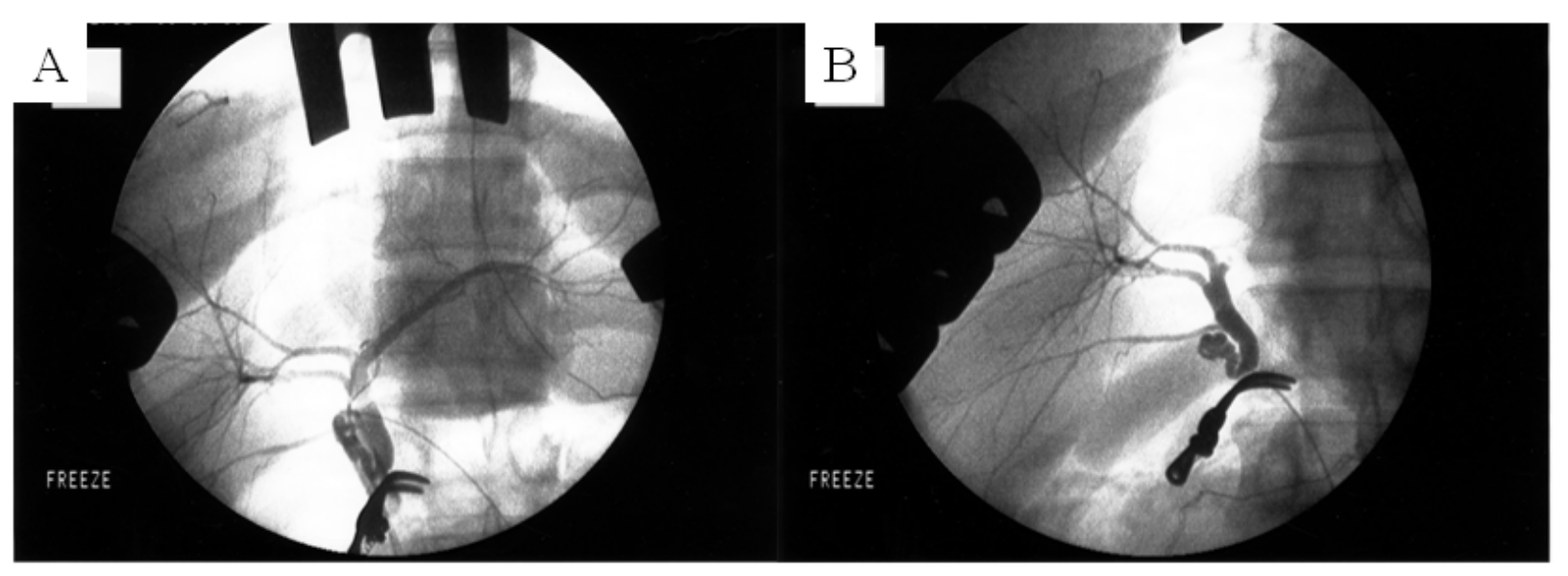

Figure 6

Bile duct division in the procurement of a left-lobe graft, in which the right anterior hepatic duct branched from the left hepatic duct (A). The bile duct was divided at an adequate point without subsequent stricture in the remnant right anterior hepatic duct (B). 
Table 1 Characteristics of donor and recipient

\begin{tabular}{|c|c|c|c|}
\hline & Group A $(n=37)$ & Group B $(n=50)$ & P value \\
\hline \multicolumn{4}{|l|}{ Donor } \\
\hline Age & $39(21-67)$ & $37(19-64)$ & NS \\
\hline Gender & Male 16 Female 21 & Male 28 Female 22 & NS \\
\hline \multicolumn{4}{|l|}{ Graft } \\
\hline RL & 28 & 23 & NS \\
\hline $\mathrm{LL}+\mathrm{CL}$ & 0 & 12 & $\mathrm{P}<0.01$ \\
\hline LL & 0 & 10 & $\mathrm{P}<0.01$ \\
\hline LLS & 9 & 0 & $\mathrm{P}<0.01$ \\
\hline RPS & 0 & 5 & $\mathrm{P}<0.01$ \\
\hline Multiple ducts in RL graft & 20/28 (71\%) & 7/23 (30\%) & $\mathrm{P}<0.01$ \\
\hline Biliary stricture & 0 & 0 & NS \\
\hline \multicolumn{4}{|l|}{ Recipient } \\
\hline Age & $41(0-65)$ & $57(11-68)$ & NS \\
\hline Gender & Male 20 Female 13 & Male 32 Female 18 & NS \\
\hline \multicolumn{4}{|l|}{ Disease } \\
\hline BA & 8 & 1 & $\mathrm{P}<0.01$ \\
\hline Viral cirrhosis & 11 & 33 & $\mathrm{P}<0.01$ \\
\hline with HCC & 5 & 27 & \\
\hline w/o HCC & 6 & 6 & \\
\hline FHF & 7 & 2 & $\mathrm{P}<0.01$ \\
\hline Others & 11 & 13 & NS \\
\hline \multicolumn{4}{|l|}{ Biliary reconstruction } \\
\hline HJ & 13 & 7 & $\mathrm{P}<0.05$ \\
\hline DD & 24 & 43 & \\
\hline Biliary stricture & 9 & 8 & NS \\
\hline Follow-up period & 79 months (52-136) & 24 months (8-48) & $\mathrm{P}<0.01$ \\
\hline
\end{tabular}

Statistical analyses were performed with Fisher's test.

$\mathrm{RL}$, right lobe; LL, left lobe; CL, caudate lobe; LLS, left lateral segment; RPS, right posterior s BA, biliary atresia; HCC, hepatocellular carcinoma; FHF, fulminant hepatic failure;

HJ, hepaticojejunostomy; DD, duct-to-duct 